\title{
Puerarin inactivates NLRP3-mediated pyroptotic cell death to alleviate cerebral ischemia/reperfusion $(I / R)$ injury through modulating the LncRNA DUXAP8/miR-223-3p axis
}

\author{
Zhenguo SHI ${ }^{\#, *}$; Qiaoyun WU*; Haiyan SHI; Songtie YING; Liang TAO \\ Ningbo Rehabilitation Hospital, Ningbo, 315000, China \\ Key words: Cerebral ischemia/reperfusion, Puerarin, LncRNA DUXAP8, miR-223-3p, NLRP3-mediated cell pyroptosis
}

\begin{abstract}
NLRP3 inflammasome-mediated cell pyroptosis aggravates the development of cerebral ischemia/reperfusion $(\mathrm{I} / \mathrm{R})$ injury, and the aim of this study is to investigate the potential utilization of the Chinese medicine, Puerarin, in treating this disease. Through conducting in vitro and in vivo experiments, the present study illustrated that Puerarin regulated LncRNA double homeobox A pseudogene 8 (DUXAP8)/miR-223-3p axis to inactivate NLRP3-mediated pyroptotic cell death, resulting in the attenuation of $\mathrm{I} / \mathrm{R}$ injury. Specifically, the cerebral $\mathrm{I} / \mathrm{R}$ injury in rat models and hypoxia/reoxygenation $(\mathrm{H} / \mathrm{R})$ in primary hippocampus neuron $(\mathrm{PHN})$ cells were inducted, which were subsequently exposed to Puerarin treatment. As expected, we validated that Puerarin suppressed cell pyroptosis and rescued cell viability in $\mathrm{I} / \mathrm{R}$ rat hippocampus tissues and H/R PHN cells. Next, through bioinformatics analysis, we noticed that miR-223-3p targeted both LncRNA DUXAP8 and NLRP3 mRNA, and both LncRNA DUXAP8 ablation and miR223-3p overexpression inactivate NLRP3-mediated cell pyroptosis to rescue cell viability in H/R PHN cells. Interestingly, we evidenced that Puerarin restrained LncRNA DUXAP8 expressions, but upregulated miR-223-3p in I/R rat tissues and H/R PHN cells, and the protective effects of Puerarin on H/R PHN cells were abrogated by overexpressing LncRNA DUXAP8 and silencing miR-223-3p. Collectively, we concluded that Puerarin regulated LncRNA DUXAP8/miR-223-3p/NLRP3 signaling cascade to attenuate I/R injury.
\end{abstract}

\section{Introduction}

Ischemic stroke accounts for approximately $87 \%$ of all stroke patients worldwide, and about 15 million people suffer from stroke each year (Benjamin et al., 2018). Currently, the main treatment therapies for cerebral ischemia includes pharmacological and mechanical reperfusion strategies (Zhang et al., 2016; Zhao et al., 2017), and researchers and clinicians agreed that timely ischemia-reperfusion strategy is effective to prevent neurological death and dysfunction (Zhang et al., 2016; Zhao et al., 2017), however, the adverse effects caused by this strategy, including intracerebral hemorrhage (Kimiwada et al., 2019; Zhang et al., 2017a), brain edema (King et al., 2018; Nakano et al., 2018), etc., still remains a serious challenge in ischemic stroke treatment. In addition, the pathogenesis mechanisms of cerebral ischemia/reperfusion (I/R) injury are very complicated, which can be attributed to

\footnotetext{
*Address correspondence to: Zhenguo Shi, diyssk0821@163.com

${ }^{\#}$ These authors contributed equally to this work

Received: 11 December 2020; Accepted: 07 July 2021
}

metabolic energy failure (Galkin, 2019; Vannucci et al., 2004), intracellular Ca2+ elevation (Sun et al., 2018; Zhang et al., 2017b), mitochondrial injury (Gong et al., 2018; Zhang et al., 2013), and cell pyroptosis-mediated inflammation (Guo et al., 2016; Zhao et al., 2019). Among those mechanisms, NLRP3 inflammasome-mediated pyroptotic cell death played an important role to aggravate $\mathrm{I} / \mathrm{R}$ injury development, and pharmacological inactivation of NLRP3 inflammasome has been proved as effective strategies to attenuate $I / R$ injury in multiple pre-clinical studies (An et al., 2019; He et al., 2019; Ma et al., 2019; Qu et al., 2019; Ye et al., 2019).

As a well-known Chinese medicine, Puerarin (7,4dihydroxyisoflavone- $8 \beta$-glucopyranoside) is one of the main bioactive isoflavone extracted from the Puerariae Lobatae Radix (Pueraria lobata (Willd.) Ohwi) root, and this drug has been recorded in the Pharmacopoeia of the People's Republic of China (2020 edition, Part I) for the treatments of cardio- and cerebrovascular diseases in China (Zhang et al., 2019d; Zhang et al., 2019e). Of note, Puerarin exerted its neuroprotective effects to alleviate cerebral $I / R$ injury in rat models (Ling et al., 2018; Liu et al., 2013; Wang et al., 2018; Zhou et al., 2014), and the available clinical data 
hinted that Puerarin was effective for the treatment of ischemic stroke in China (Liu et al., 2016). For example, JinFeng Wang et al. (2018) found that Puerarin protected rat brain against I/R injury by inactivating the AMPK-mTORULK1 pathway mediated autophagy, and Liu et al. (2013) evidenced that Puerarin activated the cholinergic antiinflammatory pathway to alleviate cerebral $\mathrm{I} / \mathrm{R}$ injury. However, as the results of the complicated nature of Chinese medicine, the underlying mechanisms are still largely unknown. Interestingly, researchers noticed that Puerarin inactivated NLRP3-meidated cell pyroptosis to regulate I/R injury (Guan et al., 2020; Wang et al., 2020). Specifically, the data provided by $\mathrm{Zi}-\mathrm{Kuang}$ Wang indicated that Puerarin regulated the SIRT1/NF-kB pathway to suppress NLRP3 inflammasome in myocardial I/R injury (Wang et al., 2020), and Guan et al. (2020) proved that Puerarin ameliorated I/R-induced retinal ganglion cell damage through regulating the TLR4/NLRP3 pathway, which enlightened us that Puerarin might protect neurons from cerebral I/R-induced death through inhibiting NLRP3mediated pyroptotic cell death.

Targeting the competing endogenous RNA (ceRNA) networks has been reported as a feasible strategy to attenuate cerebral I/R (Wan et al., 2020; Zeng et al., 2019; Zhang et al., 2019b; Zhong et al., 2019). Interestingly, recent data suggested that targeting the LncRNAs/miRNAs axis was capable of suppressing cerebral I/R development (Wan et al., 2020; Zeng et al., 2019; Zhang et al., 2019b; Zhong et al., 2019) and NLRP3-mediated cell pyroptosis (Hu et al., 2019; Yu et al., 2018). According to the previous publications, the NLRP3 mRNA could be targeted and degraded by miR-223-3p, resulting in the suppression of cell pyroptosis (Dong et al., 2019; Wan et al., 2018; Zhang et al., 2019c). In addition, upregulation of miR-223-3p suppressed necroptosis (Qin et al., 2016) and microglial M1 polarization mediated inflammation (Zhao et al., 2020) in $\mathrm{I} / \mathrm{R}$ rats, hence we selected miR-223-3p for further analysis. Moreover, LncRNA double homeobox A pseudogene 8 (DUXAP8) potentially acted as RNA sponges for miR-223$3 p$, but no literatures reported the potential regulating effects of LncRNA DUXAP8 in cerebral I/R injury or cell pyroptosis. Furthermore, multiple miRNAs could be regulated by Puerarin treatment (Zhao et al., 2020), but it was still unclear whether Puerarin regulated the LncRNA DUXAP8/miR-223-3p axis in hippocampus neural cells.

Hence, by establishing in vitro and in vivo models, the present study managed to investigate whether Puerarin attenuated the development of cerebral I/R injury through modulating the LncRNA DUXAP8/miR-223-3p/NLRP3 signaling cascade, while protein designations should be in regular capital letters.

\section{Materials and Methods}

\section{In vivo $I / R$ rat models establishment}

We bought the Sprague-Dawley (SD) rats (males, $\mathrm{N}=40$, aged 10 weeks, weighed 240-290 g) from the SiPeiFu Biotech (Beijing, China), and all the rats were fed under the specificpathogen-free (SPF) circumstances with 12-hour light-dark cycle, standard humidified atmosphere $(60 \pm 5 \%)$ and were freely accessible to water and food. The Puerarin was bought from North China Pharmaceutical Co., Ltd. (Shijiazhuang, China), and was diluted in the physiological PBS buffer at the final concentration of $25 \mathrm{mg} / \mathrm{mL}$ for further utilization at $100 \mathrm{mg} / \mathrm{kg}$ each rat according to our preliminary data (data not shown) and previous literature (Wang et al., 2018; Zhou et al., 2014). Then, the middle cerebral artery occlusion (MCAO) method was used to establish I/R rat models with the protocols as follows: the rats were intraperitoneally injected with $10 \%$ chloral hydrate at the concentration of $350 \mathrm{mg} / \mathrm{kg}$ for anesthetization, and a monofilament nylon suture was inserted into the internal carotid to generate MCAO for $90 \mathrm{~min}$, which were then removed and allowed reperfusion for $48 \mathrm{~h}$. Finally, the rat hippocampus tissues were separated and collected for further analysis. We declared that all the animal experiments were approved by the Ethics Committee of Ningbo Rehabilitation Hospital (No. 2018NBR67362).

\section{2,3,5-Tripthenyltetrazolum Chloride (TTC) staining for the measurement of infarcted volume}

TTC staining assay was performed to evaluate the infarcted area volume in rat brain in keeping with the standard experimental procedures (Zhang et al., 2019a). Briefly, the rat brains were collected and prepared as sections with $2 \mathrm{~mm}$ thickness, which were further stained with $2 \%$ TTC (Sigma, MO, USA) for $30 \mathrm{~min}$ at $37^{\circ} \mathrm{C}$, and the tissues were subsequently fixed with $4 \%$ formalin overnight at room temperature. The infarcted area was observed, and the volume was calculated.

\section{Cell culture and induction of cellular $H / R$ models}

Based on the experimental protocols provided by the previous work (Qin et al., 2020), the male newborn rat $(\mathrm{N}=5)$ were used for the isolation and purification of the primary hippocampus neuron (PHN) cells, which were maintained in the Neuro-basal Medium (Invitrogen, USA) containing poly-D-lysine (Invitrogen, USA), 2\% B27plus glucose (5 g/L) and $0.25 \%$ Glumax, in the incubator with standard culture conditions. At day 4 post-culture, the PHN cells were subjected to hypoxic stimulation to induct $\mathrm{H} / \mathrm{R}$ cellular models as previously described (Qin et al., 2020), which were further stimulated by Puerarin $(80 \mu \mathrm{M})$ for 0,24 , and $48 \mathrm{~h}$, respectively. Also, the HEK293T were obtained (ATCC, USA) and cultured in the DMEM medium (Gibco, USA) with $10 \%$ FBS (Gibco, USA).

\section{Delivery of vectors into cells}

A commercial third-party company GenePharma (Shanghai, China) was employed to design and synthesize the LncRNA DUXAP8 overexpression/downregulation vectors, and the mimic and inhibitor for miR-223-3p were obtained from GenePharma (Shanghai, China), we delivered the above vectors into the PHN cells by using the Lipofectamine 2000 transfection kit (Invitrogen, USA). At $24 \mathrm{~h}$ post-transfection, Real-Time qPCR was conducted to examine the transfection efficiency.

\section{Real-Time qPCR analysis}

Total RNA was extracted from cells and tissues by using the commercial TRIZOL reagent (Beyotime, Shanghai, China), 
which were further reversely transcribed into cDNA and quantified by performing Real-Time qPCR analysis. The detailed experimental procedures and primers could be found from the previous work (Zhao et al., 2020).

\section{Western Blot analysis}

The RIPA lysis buffer (Beyotime Biotech, Shanghai, China) was used for protein extraction and BCA kit (Beyotime Biotech, Shanghai, China) was used for protein quantification. The proteins were separated by $10 \%$ SDS-PAGE, and were transferred onto PVDF membranes (Millipore, USA). The membranes were blocked and were sequentially incubated with primary/secondary antibodies. Finally, the Western Blot Hyper HRP Substrate (TAKARA, USA) was used for protein bands visualization.

\section{Examination of cell proliferation and viability}

The primary hippocampus neuron (PHN) cells were cultured in the 96-well plates and treated with $10 \mu \mathrm{L}$ of MTT (3-[4, 5-dimethylthiazol-2-yl]-2, 5-diphenyltetrazolium bromide) solution $(5 \mathrm{mg} / \mathrm{mL})$ for $4 \mathrm{~h}$ incubation at $37^{\circ} \mathrm{C}$, and the supernatants were carefully discarded and the 96-well plates were added with dimethyl sulfoxide (DMSO). Then, the plates were vortexed, and a microplate reader (ThermoFisher, USA) was used to evaluate cell proliferation. Also, the cells were stained with trypan blue staining buffer (Merck, USA) to determine cell viability, and the cells stained with blue were regarded as dead cells.

\section{Cell apoptosis examination}

In this study, we respectively used TUNEL apoptosis kit (Beyotime, Shanghai, China) and the Annexin V-FITC/PI staining reagent (Invitrogen, USA) followed by Flow Cytometer (Becon Dickinson FACS Calibur, USA) detection to evaluate cell apoptosis in PHN cells, and the protocols were well-documented in the manufacturer's instructions.

Validation of the binding sites among LncRNA DUXAP8, miR223-3p and NLRP3 $m R N A$

The targeting sites of miR-223-3p with LncRNA DUXAP8 and 3' untranslated region (3' UTR) of NLRP3 were predicted, and the targeting sites in LncRNA DUXAP8 and NLRP3 were mutated and named as Mut-LncRNA DUXAP8 and Mut-NLRP3. In addition, their wild-type counterparts were shown as WtLncRNA DUXAP8 and Wt-NLRP3. The above sequences were cloned into the p-MIR-luciferase reporter plasmids (GenePharma, Shanghai, China), and were co-transfected with miR-NC, miR223-3p mimic and inhibitor into the HEK293T cells. At $48 \mathrm{~h}$ post-transfection, the relative luciferase activities were measured.

Enzyme linked immunosorbent assay (ELISA)

The supernatants of the PHN cells were collected, and the expression levels of IL-1 $\beta$ and IL-18 were examined by using their corresponding ELISA kit (RAPIDBIO, CA, USA) in keeping with the producers' instruction. The optical density (OD) values were measured by using a microplate reader (ThermoFisher Scientific, USA) with $450 \mathrm{~nm}$ wavelength.

Collection and analysis of the data

SPSS 18.0 software was used to analyze the data, which were shown as mean \pm SD. Specifically, the one-way ANOVA analysis was used for the comparisons among different groups. Individual experiment was repeated for at least 3 times, and ${ }^{\star} P<0.05$ was considered as statistical significance.

\section{Results}

Involvement of NLRP3-mediated cell pyroptosis in $I / R$ rat models and $H / R$ cellular models

Initially, according to the previous publications (An et al., 2019; He et al., 2019; Ma et al., 2019; Qu et al., 2019; Ye et al., 2019), we established the in vivo $\mathrm{I} / \mathrm{R}$ rat models. In Fig. 1A, the results showed that the white unstained infarcted area was lager in $\mathrm{I} / \mathrm{R}$ rats but not their normal counterparts $(P<0.01)$. Next, the hippocampus tissues were collected from the brain of the rats for further analysis. The hippocampus tissues were stained by TUNEL, and the results in Fig. $1 \mathrm{~B}$ showed that $\mathrm{I} / \mathrm{R}$ treatment significantly increased the TUNEL-positive apoptotic cell ratio in rat tissues $(P<0.01)$, and the data in Suppl. Fig. S1A supported that $\mathrm{I} / \mathrm{R}$ significantly increased neurological deficit score in rats, as determined by using the Longa scoring system. Consistently, the expression levels of cleaved Caspase- 3 and Bax were increased $(P<0.05$, Fig. $1 C)$, while the Cyclin D1 and CDK2 were increased $(P<0.05$, Fig. $1 D)$ in rat tissues, suggesting that $\mathrm{I} / \mathrm{R}$ suppressed hippocampus viability in vivo. Interestingly, as shown in Fig. $1 \mathrm{E}$, we noticed that $\mathrm{I} / \mathrm{R}$ also upregulated NLRP3 and ASC to induce cell pyroptosis in rat hippocampus tissues $(P<0.05)$. In addition, the $\mathrm{H} / \mathrm{R}$ models in the PHN cells were inducted to simulate the real conditions of $\mathrm{I} / \mathrm{R}$ injury in vitro. As expected, cell proliferation (Fig. 1F) and viability (Fig. $1 \mathrm{G}$ ) were significantly decreased in the PHN cells treated with $\mathrm{H} / \mathrm{R}$, in contrast with the normal PHN cells $(P<0.05)$. Also, the levels of NLRP3, ASC, IL-1 $\beta$ and IL-18 in PHN cells $(P<0.05$, Fig. $1 \mathrm{H})$ and their supernatants $(P<0.05$, Figs. $1 \mathrm{I}$ and $1 \mathrm{~J}$ ) were also increased by $\mathrm{H} / \mathrm{R}$.

Puerarin inactivated NLRP3-mediated pyroptotic cell death to attenuate $I / R$ and $H / R$ injury in vivo and in vitro

As previously described (Guan et al., 2020; Wang et al., 2020), Puerarin exerted its neuroprotective effects during I/R injury, which were validated by our following experiments in Figs. 2A-2I and Suppl. Fig. S1A and S1B. Specifically, the rats were divided into four groups, including Sham, I/R group, Puerarin $(100 \mathrm{mg} / \mathrm{kg})$ group, and $\mathrm{I} / \mathrm{R}+$ Puerarin $(100 \mathrm{mg} / \mathrm{kg})$ group, and the results in Suppl. Figs. S1A and S1B showed that Puerarin decreased neurological deficit score and infarcted area volume in $\mathrm{I} / \mathrm{R}$ rats. Further Western Blot analysis results showed that Puerarin downregulated cleaved Caspase- 3 and $\operatorname{Bax}(P<0.05$, Fig. 2A), and upregulated Cyclin D1 and CDK2 $(P<0.05$, Fig. $2 \mathrm{~B})$ in rat hippocampus tissues. Besides, NLRP3 and ASC expressions were also downregulated by Puerarin treatment $(P<0.05$, Fig. $2 C)$. In addition, our in vivo results were supported by the following in vitro experiments, and the results suggested that Puerarin rescued cell proliferation $(P<0.05$, Fig. $2 \mathrm{D})$ and viability $(P<0.05$, Fig. 2E) in PHN cells treated with $\mathrm{H} / \mathrm{R}$. Also, we evidenced that Puerarin attenuated cell apoptosis in H/R-treated PHN cells $(P<0.05$, Fig. $2 \mathrm{~F})$. Finally, the aggravating effects of $H / R$ on NLRP3, ASC, IL-1 $\beta$ and IL-18 in the PHN cells (Fig. 2G) and their supernatants (Figs. $2 \mathrm{H}$ and $2 \mathrm{I}$ ) were abrogated by 


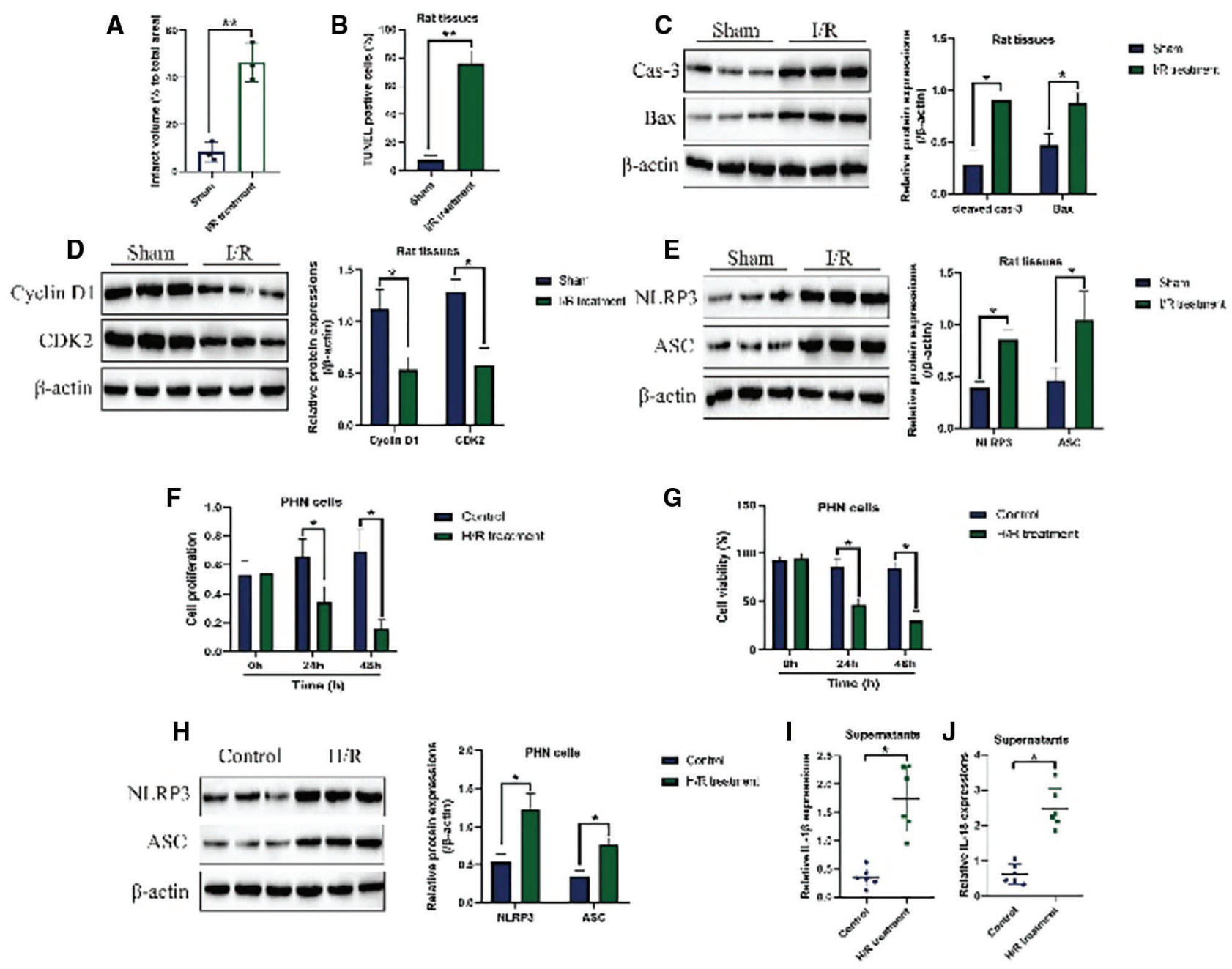

FIGURE 1. NLRP3-mediated cell pyroptosis was closely associated with in vivo I/R injury in rat hippocampus tissues and in vitro H/R injury in PHN cells. (A) The rat brain was obtained, and the white unstained infarcted area was observed and recorded. (B) Cell apoptosis in rat tissues was determined by TUNEL staining assay. Western Blot was used to examine (C) apoptosis, (D) cell cycle, (E) pyroptosis associated biomarkers. The cellular functions, including $(\mathrm{F})$ cell proliferation and $(\mathrm{G})$ viability were respectively examined. The expression status of pyroptosis markers were examined in the $(\mathrm{H})$ cells and $(\mathrm{I}-\mathrm{J})$ supernatants. Each experiment was repeated three times; ${ }^{\star} P<0.05,{ }^{\star \star} P<0.01$.

co-treating cells with Puerarin $(P<0.05)$, indicating that Puerarin attenuated I/R- and H/R-induced hippocampus injury in vivo and in vitro.

Puerarin negatively regulated NLRP3 through upregulating $m i R-223-3 p$

NLRP3 could be negatively modulated by its upstream transcriptional regulators, including miRNAs (Hu et al., 2019; Yu et al., 2018), which were closely associated with $\mathrm{I} / \mathrm{R}$ injury and could be modulated by Puerarin. Therefore, we speculated that Puerarin might regulate NLRP3-mediated cell pyroptosis through modulating miRNAs. To validate this hypothesis, ten miRNAs that potentially regulated NLRP3 was selected, and we found that only miR-223-3p, but not other types of miRNAs, could be downregulated by $\mathrm{H} / \mathrm{R}$, but it was upregulated by Puerarin in PHN cells $(P<0.05$, Fig. 3A). Consistently, the results in Fig. 3B showed that $I / R$ treatment also decreased miR-223-3p levels in rat hippocampus tissues, which could be significantly increased by Puerarin $(P<0.05$, Fig. 3B). Next, the targeting sites of miR-223-3p with 3' untranslated regions of NLRP3 mRNA were predicted, and the targeting sites in NLRP3 mRNA were subsequently mutated (Fig. 3C). As shown in Fig. 3D, the dual-luciferase reporter gene system assay validated that miR223-3p bound to the 3'UTR of NLRP3 mRNA. Subsequently, miR-223-3p was downregulated and upregulated in the $H / R$ PHN cells (Fig. 3E), and the following Real-Time qPCR and Western Blot analysis results evidenced that miR-223-3p negatively regulated NLRP3 expressions in H/R treated PHN cells at both transcriptional $(P<0.05$, Fig. $3 \mathrm{~F})$ and translational (Fig. 3G) levels. In addition, the inhibiting effects of Puerarin on H/R-induced cell pyroptosis in PHN cells were partly reversed by silencing miR-223-3p $(P<0.05$, Figs. 3H and 3I).

The regulating mechanisms of LncRNA DUXAP8-miR-223-3pNLRP3 axis in vitro

Given that there existed LncRNAs-miRNAs-mRNAs ceRNA networks, and LncRNAs had also been reported to participate in the regulation of I/R injury (Wan et al., 2020; Zeng et al., 2019; Zhang et al., 2019b; Zhong et al., 2019), we next screened out that the miR-223-3p/NLRP3 pathway could be modulated by LncRNA DUXAP8 in PHN cells in Figs. 4A-4I. Specifically, seven LncRNAs containing the 

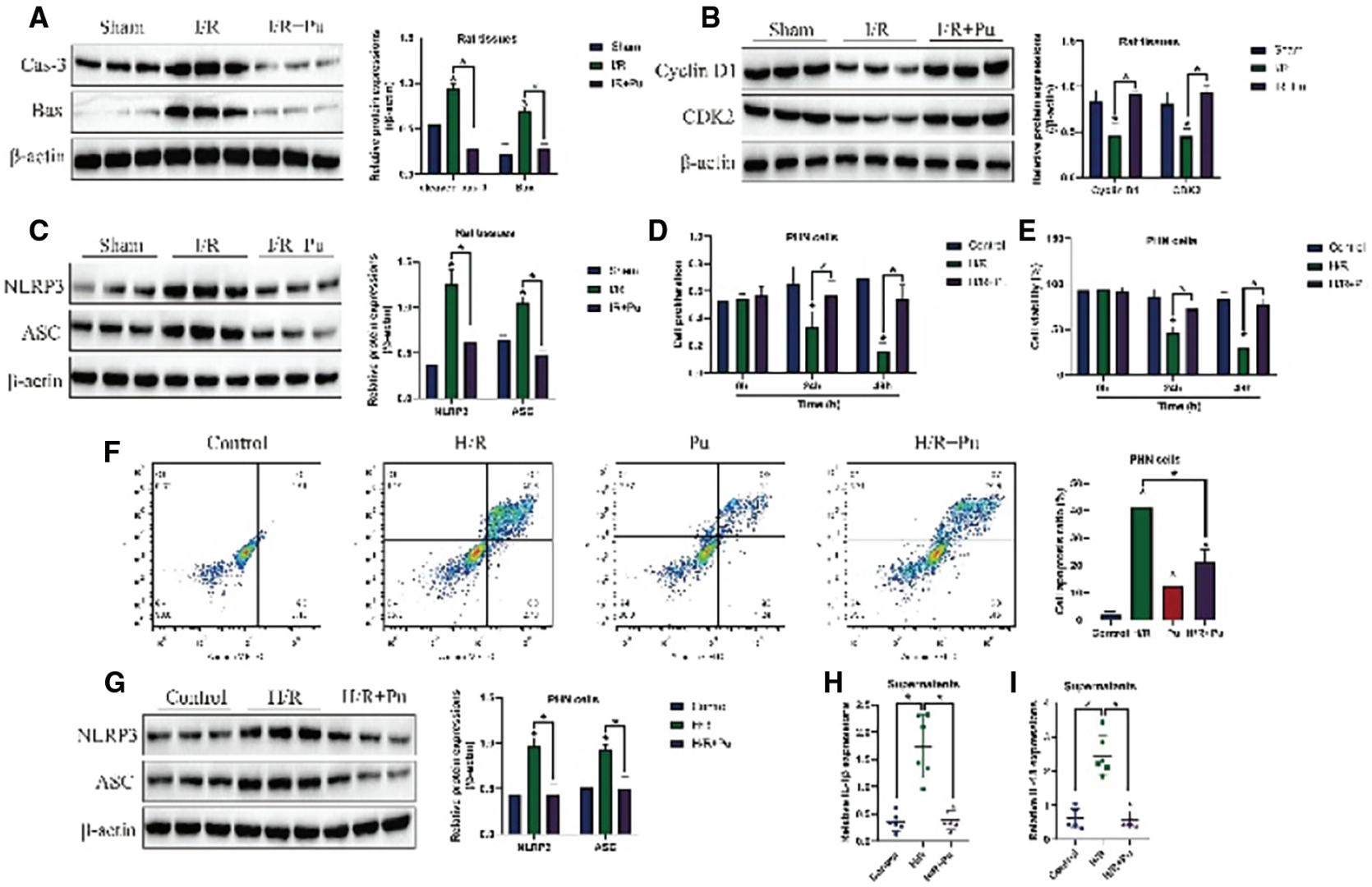

FIGURE 2. Puerarin restrained $\mathrm{I} / \mathrm{R}$ and $\mathrm{H} / \mathrm{R}$ injury for potential protection. (A) Cell apoptosis, (B) cell cycle and (C) pyroptosis associated proteins were examined. The cellular biological functions, such as (D) cell proliferation and (E) viability were determined. (F) Cell apoptosis ratio was measured by flow cytometer. (G-I) The pyroptosis associated biomarkers were measured ("Pu" indicated Puerarin treatment). Each experiment was repeated three times; ${ }^{\star} P<0.05$.
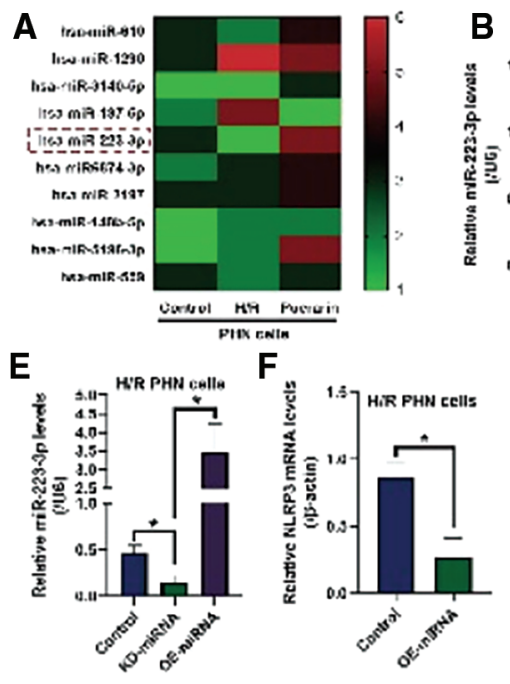
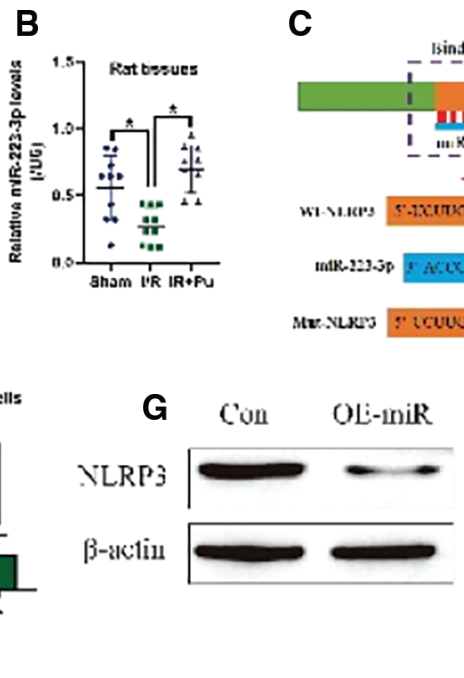

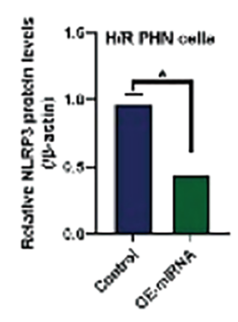

C
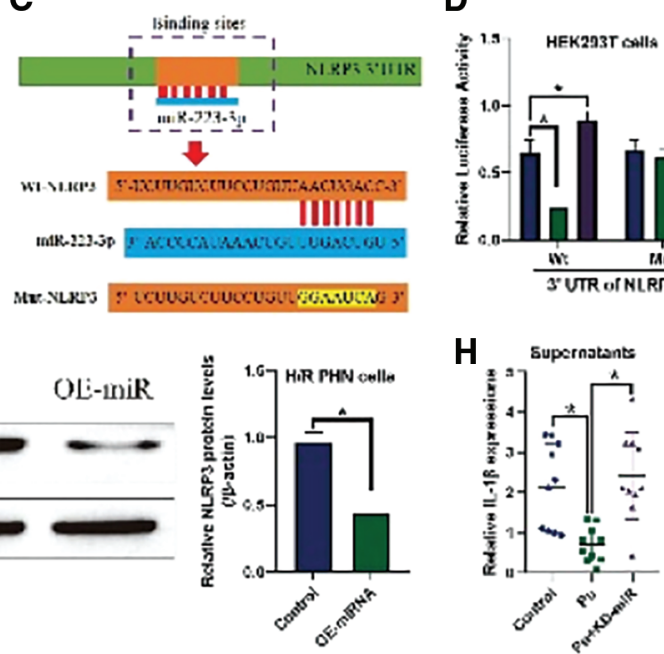

a nirner,

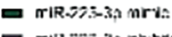
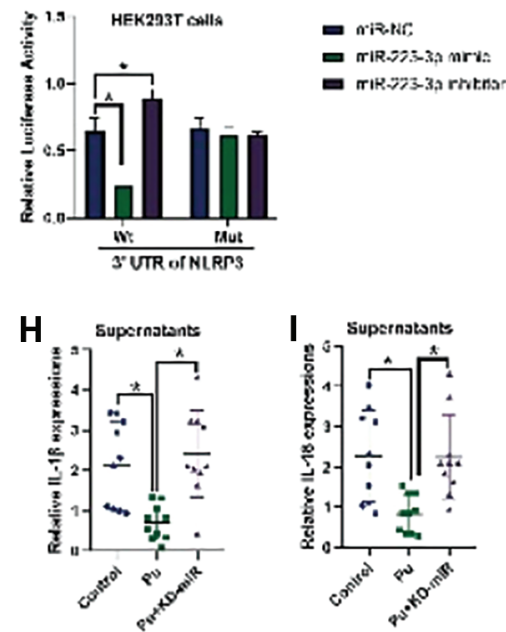

FIGURE 3. The regulatory mechanisms of miR-223-3p and NLRP3. The aberrant expressions of miRNAs were screened by Real-Time qPCR in the (A) PHN cells and (B) rat hippocampus tissues. The targeting sites of miR-223-3p and NLRP3 mRNA were (C) predicted and (D) validated. (E-G) NLRP3 could be negatively regulated by miR-223-3p. (H-I) The pro-pyroptosis cytokines in the supernatants were measured by ELISA (“OE-miR” represented miR-223-3p overexpression). Each experiment was repeated three times; ${ }^{\star} P<0.05$.

binding sites with miR-223-3p were selected, and further RealTime qPCR results screened out that LncRNA DUXAP8 was upregulated by $\mathrm{H} / \mathrm{R}$ treatment in PHN cells, which could be significantly downregulated by Puerarin $(P<0.05$, Fig. $4 \mathrm{~A})$. Consistently, we evidenced that LncRNA DUXAP8 was also upregulated by $I / R$ and downregulated by Puerarin in rat hippocampus tissues $(P<0.05$, Fig. 4B). Hence, the LncRNA DUXAP8 was chosen for further analysis. The targeting sites of miR-223-3p with LncRNA DUXAP8 (Fig. 4C) were predicted, which were validated by the following experiments (Fig. 4D). Next, the overexpression and silencing vectors for LncRNA DUXAP8 were delivered 

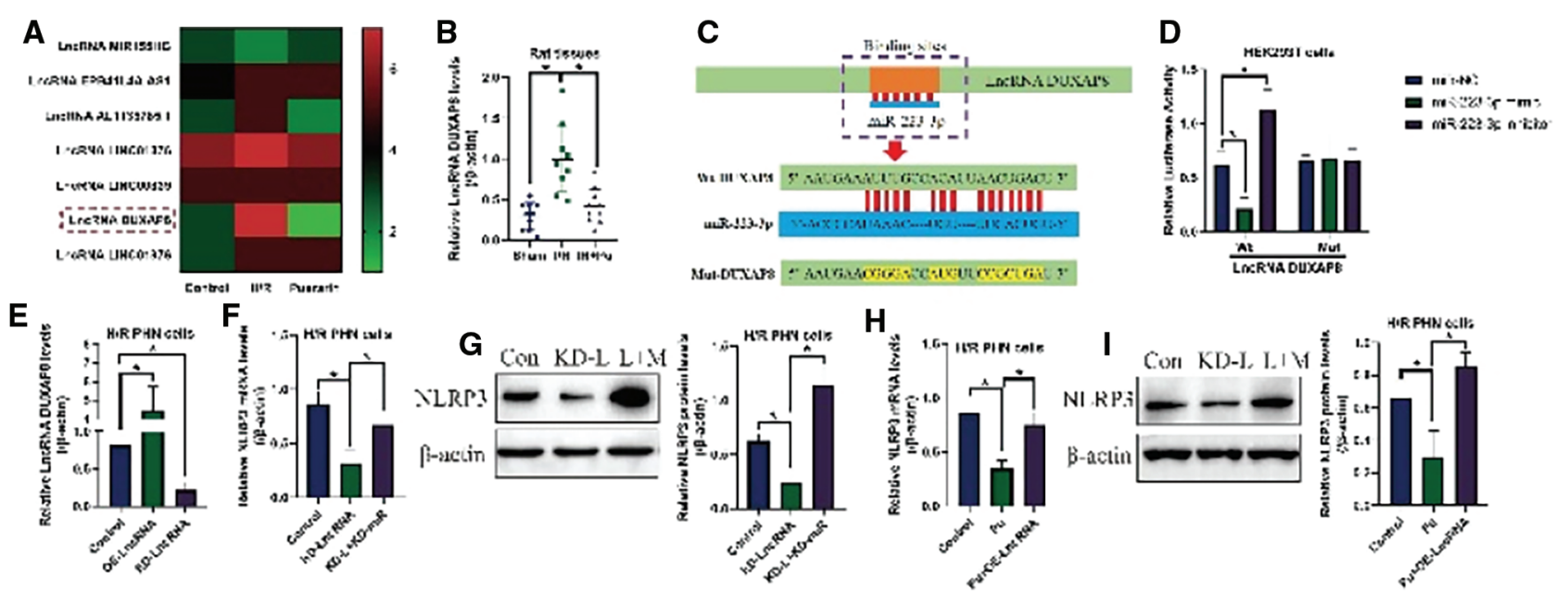

FIGURE 4. LncRNA DUXAP8 positively regulated NLRP3 in the PHN cells by sponging miR-223-3p. (A) Multiple LncRNAs were screened, and (B) LncRNA DUXAP8 was significantly influenced by I/R treatment. (C) Prediction and (D) validation of miR-223-3p-LncRNA DUXAP8 binding sites. (E) LncRNA DUXAP8 was manipulated, and (F, G) the regulating effects of LncRNA DUXAP8 on NLRP3 expressions were examined. (H-I) Puerarin suppressed NLRP3 in PHN cells by regulating the LncRNA DUXAP8/miR-223-3p axis ("Con" indicated Control, "KD-L" suggested knockdown of LncRNA DUXAP8, and "L+M" represented knockdown of LncRNA DUXAP8 plus miR-223-3p ablation). Each experiment was repeated three times; ${ }^{\star} P<0.05$.

into the H/R PHN cells (Fig. 4E), by performing Real-Time qPCR and Western Blot, we found that knock-down of LncRNA DUXAP8 decreased the mRNA and protein levels of NLRP3 in H/R-treated PHN cells, which were reversed by silencing miR-223-3p $(P<0.05$, Figs. $4 \mathrm{~F}$ and $4 \mathrm{G})$. In addition, we found that Puerarin decreased the expression levels of NLRP3 in H/R-treated PHN cells, which were increased by upregulating LncRNA DUXAP8 ( $P<0.05$, Figs. 4H and 4I).
A

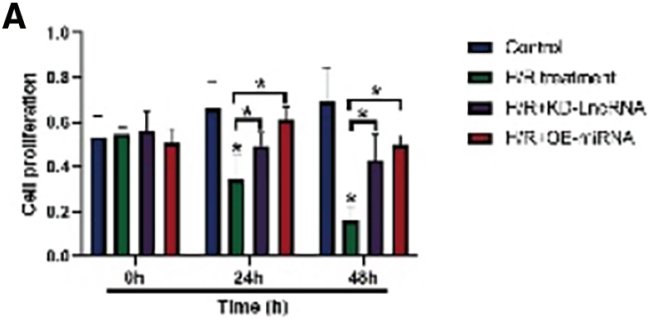

B

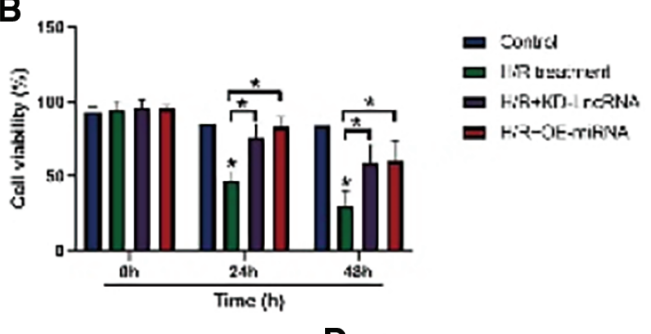

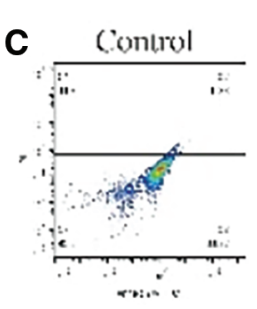
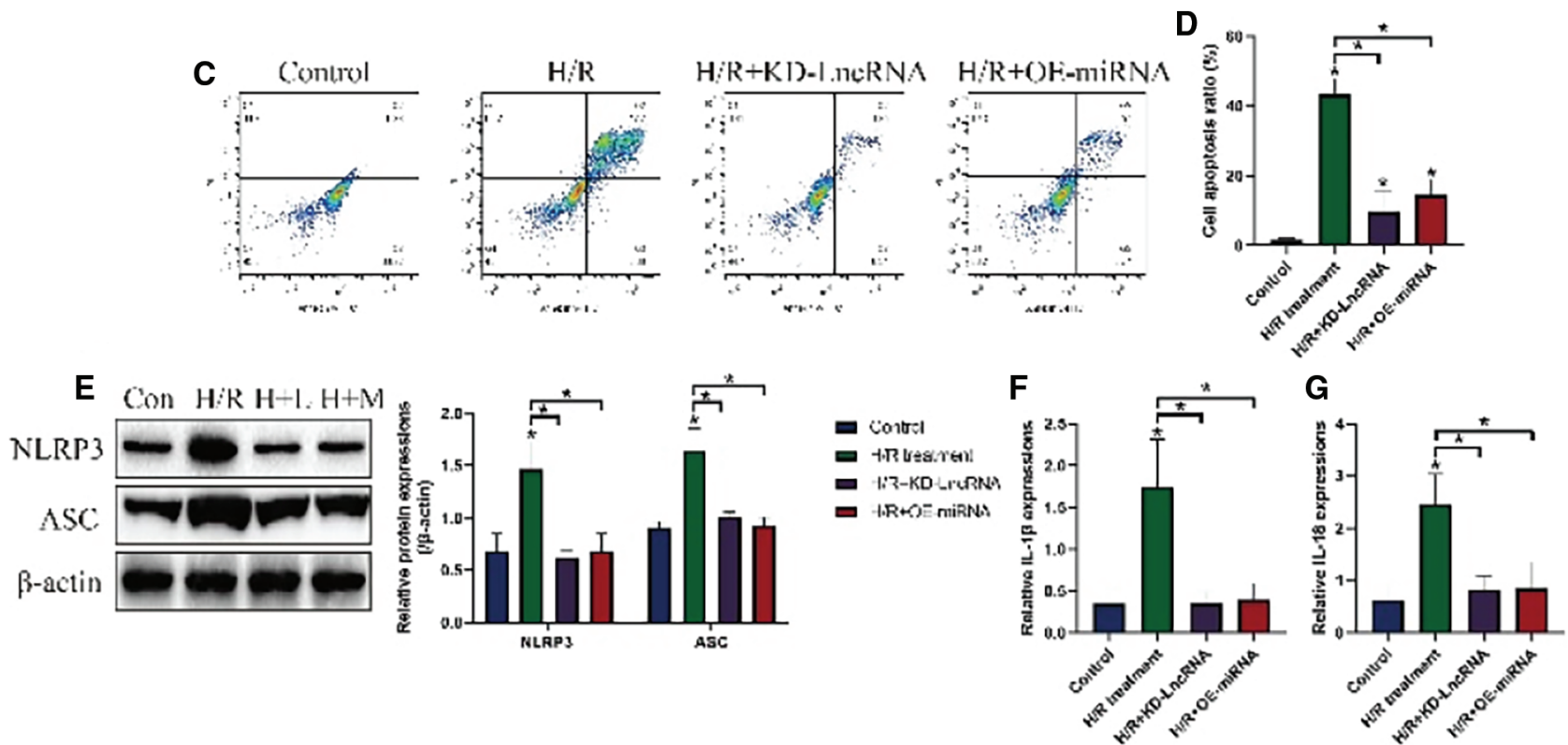

FIGURE 5. Targeting the LncRNA DUXAP8/miR-223-3p axis rescued cell functions in PHN cells. (A) Cell proliferation and (B) viability were respectively determined. (C-D) Flow cytometer was used to detect the Annexin V-FITC and PI-positive apoptotic cells. The pyroptosis indicators were examined in both (E) PHN cells and (F-G) their supernatants ("Con" represented Control, "H/R" indicated H/R treatment, "H+L" indicated H/R treatment plus LncRNA DUXAP8 knockdown, and "H+M" represented H/R treatment plus miR-223-3p overexpression). Each experiment was repeated three times; ${ }^{\star} P<0.05$. 
Targeting the LncRNA DUXAP8/miR-223-3p axis rescued cell viability in $H / R$ PHN cells

The above data encouraged us to assure whether the LncRNA DUXAP8/miR-223-3p axis involved in regulating the development of I/R-induced injury in vitro. To achieve this, LncRNA DUXAP8 was silenced $(P<0.05$, Fig. $4 \mathrm{E})$, while miR-223-3p was overexpressed $(P<0.05$, Fig. $3 E)$ in $H / R$ PHN cells, and the results showed that both LncRNA DUXAP8 ablation and miR-223-3p overexpression restored cell proliferation (Fig. 5A) and viability (Fig. 5B) in H/Rtreated PHN cells $(P<0.05)$. In addition, the cell apoptosis examination assay results indicated that $H / R$ treatment significantly promoted cell apoptosis in PHN cells, which were reversed by ablating LncRNA DUXAP8 and overexpressing miR-223-3p $(P<0.05$, Figs. 5C and 5D). Further results validated that silencing of LncRNA DUXAP8 and overexpression of miR-223-3p also downregulated NLRP3, ASC, IL-1 $\beta$ and IL-18 to inactivate NLRP3mediated cell pyroptosis in H/R PHN cells $(P<0.05$, Fig. 5E) and the supernatants $(P<0.05$, Figs. $5 \mathrm{~F}$ and $5 \mathrm{G})$.
Puerarin promoted cell survival in $H / R$ PHN cells via the LncRNA DUXAP8/miR-223-3p axis

Finally, we validated that Puerarin regulated the LncRNA DUXAP8/miR-223-3p axis to exert its neuroprotective effects on H/R PHN cells. Specifically, the H/R PHN cells were pre-transfected with LncRNA DUXAP8 overexpression vectors $(P<0.05$, Fig. $4 \mathrm{E})$ and miR-223-3p ablation vectors $(P<0.05$, Fig. 3E), and were divided into four groups, including Control, Puerarin group, Puerarin + OE- LncRNA DUXAP8 group, and Puerarin + KD-miR-223-3p group. The results showed that Puerarin rescued cell proliferation in H/R PHN cells, which were abrogated by overexpressing LncRNA DUXAP8 and downregulating miR-223-3p $(P<$ 0.05 , Fig. $6 \mathrm{~A}$ ). Consistently, further data validated that both LncRNA DUXAP8 overexpression and miR-223-3p ablation abrogated the promoting effects of Puerarin on cell viability in $\mathrm{H} / \mathrm{R}$ PHN cells $(P<0.05$, Fig. 6B). Furthermore, we evidenced that Puerarin also suppress cell apoptosis in $H / R$ PHN cells by targeting the LncRNA DUXAP8/miR-223-3p axis $(P<0.05$, Fig. $6 \mathrm{C})$. The above results suggested that
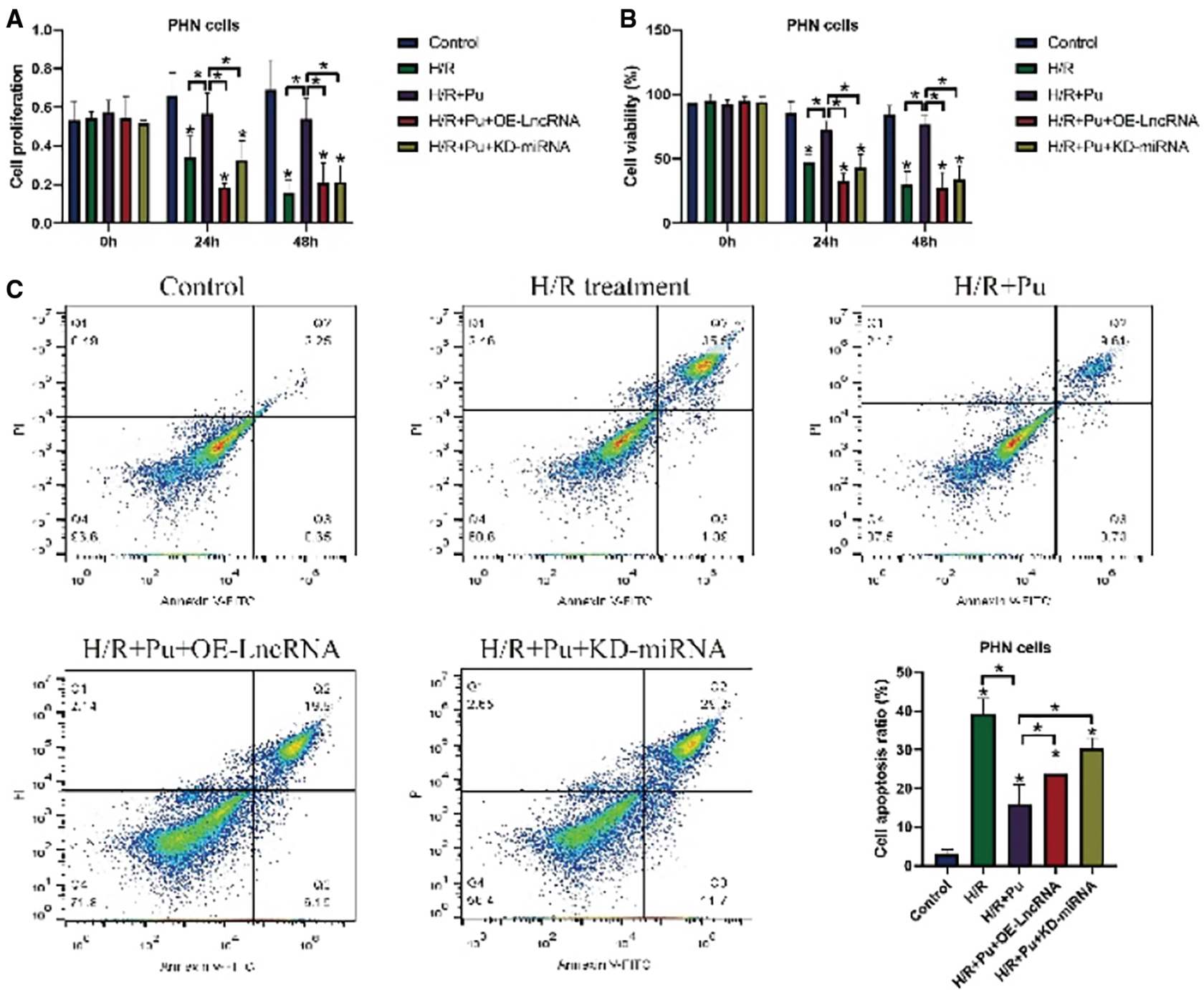

FIGURE 6. Puerarin regulated the LncRNA DUXAP8/miR-223-3p axis to attenuate H/R injury in the PHN cells in vitro. PHN cell (A) proliferation and (B) viability were evaluated. (C) Examination of cell apoptosis by flow cytometer ("Pu" indicated Puerarin, OE-LncRNA represented LncRNA DUXAP8 overexpression, and "KD-miRNA" suggested miR-223-3p ablation). Each experiment was repeated three times; ${ }^{\star} P<0.05$. 
Puerarin regulated the LncRNA DUXAP8/miR-223-3p axis to attenuate cell death in H/R PHN cells in vitro.

\section{Discussion}

Ischemic stroke seriously degrades the life quality of human beings worldwide (Benjamin et al., 2018), and timely pharmacological and mechanical reperfusion are proved as effective strategies for its treatment (Zhang et al., 2016; Zhao et al., 2017). However, recent data indicated that cerebral ischemia/reperfusion (I/R) injury caused serious side effects, such as hemorrhage (Kimiwada et al., 2019; Zhang et al., 2017a), brain edema (King et al., 2018; Nakano et al., 2018), etc., were still a great obstacle for this treatment therapy in clinic, and uncovering the molecular mechanisms of cerebral $\mathrm{I} / \mathrm{R}$ injury pathogenesis and development of novel therapeutic drugs to attenuate $I / R$ injury might help to solve these problems. This study found that Puerarin regulated a novel LncRNA DUXAP8/miR-223-3p/NLRP3 inflammasomemediated cell pyroptosis to alleviate cerebral I/R injury. Also, previous publications indicated that NLRP3-meidated cell pyrotosis contributed to the development cerebral I/R injury (An et al., 2019; He et al., 2019; Ma et al., 2019; Qu et al., 2019; Ye et al., 2019), which were validated by our present work. Specifically, the cerebral I/R rat models and H/R PHN cell models were established, and we found that the expression levels of pyroptosis associated signatures were upregulated in both I/R rats and H/R PHN cells, in contrast with their normal counterparts, suggesting that cell pyroptosis occurred and contributed to cerebral I/R injury.

Puerarin a well-known Chinese medicine extracted from the Kudzu (Pueraria montana var. lobata) root and has been used as therapeutic drug for multiple cardio- and cerebrovascular diseases in China (Zhang et al., 2019d; Zhang et al., 2019e). Of note, recent publications evidenced that Puerarin also exerted its neuroprotective effects to alleviate cerebral I/R injury in rat models (Ling et al., 2018; Liu et al., 2013; Wang et al., 2018; Zhou et al., 2014), and our experiments supported that Puerarin treatment increased cell survival and suppressed cell apoptosis in both $\mathrm{I} / \mathrm{R}$ rat hippocampus tissues and $\mathrm{H} / \mathrm{R}$ PHN cells, implying that Puerarin could be utilized as a therapeutic agent for cerebral I/R injury. Interestingly, previous data suggested that Puerarin inactivated NLRP3-meidated cell pyroptosis to regulate I/R injury (Guan et al., 2020; Wang et al., 2020), and our data showed that Puerarin restrained and inactivated cell pyroptosis in I/R rat models and H/R PHN cell models.

Targeting the LncRNAs-miRNAs-mRNAs ceRNA networks is effective to treat cerebral $\mathrm{I} / \mathrm{R}$ injury (Wan et al., 2020; Zeng et al., 2019; Zhang et al., 2019b; Zhong et al., 2019) and NLRP3-mediated pyroptotic cell death (Hu et al., 2019; Yu et al., 2018), and we identified a novel LncRNA DUXAP8/miR223-3p axis that regulated NLRP3-mediated cell pyroptosis to regulate $\mathrm{H} / \mathrm{R}$-induced $\mathrm{PHN}$ cell injury in vitro. Mechanistically, miR-223-3p targeted both LncRNA DUXAP8 and NLRP3 mRNA, and LncRNA DUXAP8 positively regulated NLRP3 expressions by acting as RNA sponge for miR-223-3p, which were partly supported by the previous studies (Dong et al., 2019; Wan et al., 2018; Zhang et al., 2019c) and validated that LncRNA DUXAP8-miR-223-3p-NLRP3 ceRNA networks existed in the H/R PHN cell models. In addition, previous data suggested that miR-223-3p attenuated cerebral I/R injury (Qin et al., 2016; Zhao et al., 2020), and we evidenced that both LncRNA DUXAP8 ablation and miR-223-3p overexpression promoted cell survival in $H / R$ PHN cells. Furthermore, multiple miRNAs could be regulated by Puerarin (Zhao et al., 2020), and we first identified that Puerarin downregulated LncRNA DUXAP8 and upregulated miR-223-3p in PHN cells. Also, our data showed that the protective effects of Puerarin on H/R PHN cells were abrogated by upregulating LncRNA DUXAP8 and silencing miR-223-3p, implying that Puerarin regulated the LncRNA DUXAP8/miR-223-3p axis to attenuate H/R-induced cell death in PHN cells.

\section{Conclusions}

Taken together, the present study evidenced that Puerarin regulated the LncRNA DUXAP8/miR-223-3p axis to inactivate NLRP3 inflammasome-mediated pyroptotic cell death, resulting in the alleviation of cerebral I/R injury.

Availability of Data and Materials: All the involved data had been included in this manuscript, and the original raw data could be obtained from the corresponding author.

Author Contribution: The authors confirm contribution to the paper as follows: study conception and design: Zhenguo Shi, Qiaoyun $\mathrm{Wu}$; data collection: Haiyan Shi; analysis and interpretation of results: Songtie Ying; draft manuscript preparation: Zhenguo Shi, Qiaoyun Wu. All authors reviewed the results and approved the final version of the manuscript.

Ethics Approval: We declared that all the animal experiments were approved by the Ethics Committee of Ningbo Rehabilitation Hospital (No. 2018NBR67362).

Funding Statement: The present study was financially supported by the Project of Scientific Research Fund of Traditional Chinese Medicine of Zhejiang Province (No. 2020ZB230).

Conflicts of Interest: The authors declare that they have no conflicts of interest to report regarding the present study.

\section{References}

An P, Xie J, Qiu S, Liu Y, Wang J et al. (2019). Hispidulin exhibits neuroprotective activities against cerebral ischemia reperfusion injury through suppressing NLRP3-mediated pyroptosis. Life Sciences 232: 116599. DOI 10.1016/j. lfs.2019.116599.

Benjamin EJ, Virani SS, Callaway CW, Chamberlain AM, Chang AR et al. (2018). Heart disease and stroke statistics-2018 update: A report from the American Heart Association. Circulation 137: 116. DOI 10.1161/CIR.0000000000000558.

Dong HC, Li PN, Chen CJ, Xu X, Zhang H et al. (2019). Sinomenine attenuates cartilage degeneration by regulating miR-223-3p/ NLRP3 inflammasome signaling. Inflammation 42: 12651275. DOI 10.1007/s10753-019-00986-3.

Galkin A (2019). Brain ischemia/reperfusion injury and mitochondrial complex I damage. Biochemistry 84: 1411-1423. 
Gong Z, Pan J, Shen Q, Li M, Peng Y (2018). Mitochondrial dysfunction induces NLRP3 inflammasome activation during cerebral ischemia/reperfusion injury. Journal of Neuroinflammation 15: 78. DOI 10.1186/s12974-018-1282-6.

Guan L, Li C, Zhang Y, Gong J, Wang G et al. (2020). Puerarin ameliorates retinal ganglion cell damage induced by retinal ischemia/reperfusion through inhibiting the activation of TLR4/NLRP3 inflammasome. Life Sciences 256: 117935. DOI 10.1016/j.lfs.2020.117935.

Guo Z, Yu S, Chen X, Ye R, Zhu W, Liu X (2016). NLRP3 is involved in ischemia/reperfusion injury. CNS \& Neurological Disorders-Drug Targets 15: 699-712. DOI 10.2174/1871527315666160321111829.

He Q, Li Z, Meng C, Wu J, Zhao Y, Zhao J (2019). Parkin-dependent mitophagy is required for the inhibition of ATF4 on NLRP3 inflammasome activation in cerebral ischemia-reperfusion injury in rats. Cells 8: 897. DOI 10.3390/cells8080897.

Hu J, Wu H, Wang D, Yang Z, Dong J (2019). LncRNA ANRIL promotes NLRP3 inflammasome activation in uric acid nephropathy through miR-122-5p/BRCC3 axis. Biochimie 157: 102-110. DOI 10.1016/j.biochi.2018.10.011.

Kimiwada T, Hayashi T, Takahashi M, Shirane R, Tominaga T (2019). Progressive cerebral ischemia and intracerebral hemorrhage after indirect revascularization for a patient with cerebral proliferative angiopathy. Journal of Stroke and Cerebrovascular Diseases 28: 853-858. DOI 10.1016/j. jstrokecerebrovasdis.2018.11.021.

King ZA, Sheth KN, Kimberly WT, Simard JM (2018). Profile of intravenous glyburide for the prevention of cerebral edema following large hemispheric infarction: Evidence to date. Drug Design, Development and Therapy 12: 2539-2552. DOI 10.2147/DDDT.

Ling C, Liang J, Zhang C, Li R, Mou Q et al. (2018). Synergistic effects of salvianolic acid $\mathrm{B}$ and puerarin on cerebral ischemia reperfusion injury. Molecules 23: 564. DOI 10.3390/molecules23030564.

Liu B, Tan Y, Wang D, Liu M (2016). Puerarin for ischaemic stroke. Cochrane Database of Systematic Reviews 2: Cd004955. DOI 10.1002/14651858.

Liu X, Mei Z, Qian J, Zeng Y, Wang M (2013). Puerarin partly counteracts the inflammatory response after cerebral ischemia/ reperfusion via activating the cholinergic anti-inflammatory pathway. Neural Regeneration Research 8: 3203-3215.

Ma C, Wang X, Xu T, Yu X, Zhang S et al. (2019). Qingkailing injection ameliorates cerebral ischemia-reperfusion injury and modulates the AMPK/NLRP3 Inflammasome Signalling pathway. BMC Complementary and Alternative Medicine 19: 161. DOI 10.1186/s12906-019-2703-5.

Nakano T, Nishigami C, Irie K, Shigemori Y, Sano K et al. (2018). Goreisan prevents brain edema after cerebral ischemic stroke by inhibiting aquaporin 4 upregulation in mice. Journal of Stroke and Cerebrovascular Diseases 27: 758-763. DOI 10.1016/j.jstrokecerebrovasdis.2017.10.010.

Qin D, Wang X, Li Y, Yang L, Wang R et al. (2016). MicroRNA-223$5 p$ and $-3 p$ cooperatively suppress necroptosis in ischemic/ reperfused hearts. Journal of Biological Chemistry 291: 20247-20259. DOI 10.1074/jbc.M116.732735.

Qin J, Ma Q, Ma D (2020). Low-dose sevoflurane attenuates cardiopulmonary bypass (CPB)-induced postoperative cognitive dysfunction (POCD) by regulating hippocampus apoptosis via PI3K/AKT pathway. Current Neurovascular Research 17: 232240. DOI $10.2174 / 1567202617666200513085403$.

Qu XY, Zhang YM, Tao LN, Gao H, Zhai JH et al. (2019). XingNaoJing injections protect against cerebral ischemia/reperfusion injury and alleviate blood-brain barrier disruption in rats, through an underlying mechanism of NLRP3 inflammasomes suppression. Chinese Journal of Natural Medicines 17: 498505. DOI 10.1016/S1875-5364(19)30071-8.

Sun B, Ou H, Ren F, Huan Y, Zhong T et al. (2018). Propofol inhibited autophagy through $\mathrm{Ca}(2+) / \mathrm{CaMKK} \beta / \mathrm{AMPK} / \mathrm{mTOR}$ pathway in OGD/R-induced neuron injury. Molecular Medicine 24: 138. DOI 10.1186/s10020-018-0054-1.

Vannucci RC, Towfighi J, Vannucci SJ (2004). Secondary energy failure after cerebral hypoxia-ischemia in the immature rat. Journal of Cerebral Blood Flow \& Metabolism 24: 10901097. DOI 10.1097/01.WCB.0000133250.03953.63.

Wan L, Yuan X, Liu M, Xue B (2018). miRNA-223-3p regulates NLRP3 to promote apoptosis and inhibit proliferation of hep3B cells. Experimental and Therapeutic Medicine 15: 2429-2435.

Wan P, Su W, Zhang Y, Li Z, Deng C et al. (2020). LncRNA H19 initiates microglial pyroptosis and neuronal death in retinal ischemia/reperfusion injury. Cell Death \& Differentiation 27: 176-191. DOI 10.1038/s41418-019-0351-4.

Wang JF, Mei ZG, Fu Y, Yang SB, Zhang SZ et al. (2018). Puerarin protects rat brain against ischemia/reperfusion injury by suppressing autophagy via the AMPK-mTOR-ULK1 signaling pathway. Neural Regeneration Research 13: 989998. DOI 10.4103/1673-5374.233441.

Wang ZK, Chen RR, Li JH, Chen JY, Li W et al. (2020). Puerarin protects against myocardial ischemia/reperfusion injury by inhibiting inflammation and the NLRP3 inflammasome: The role of the SIRT1/NF-kB pathway. International Immunopharmacology 89: 107086. DOI 10.1016/j.intimp.2020.107086.

Ye Y, Jin T, Zhang X, Zeng Z, Ye B et al. (2019). Meisoindigo protects against focal cerebral ischemia-reperfusion injury by inhibiting NLRP3 inflammasome activation and regulating microglia/macrophage polarization via TLR4/NF- $\mathrm{BB}$ signaling pathway. Frontiers in Cellular Neuroscience 13: 574. DOI 10.3389/fncel.2019.00553.

Yu SY, Dong B, Tang L, Zhou SH (2018). LncRNA MALAT1 sponges miR-133 to promote NLRP3 inflammasome expression in ischemia-reperfusion injured heart. International Journal of Cardiology 254: 50. DOI 10.1016/j.ijcard.2017.10.071.

Zhang Y, Chen WA, Huang SS, Wang HM (2016). Protective effects of mangiferin on cerebral ischemia-reperfusion injury and its mechanisms. European Journal of Pharmacology 771: 145151. DOI 10.1016/j.ejphar.2015.12.003.

Zeng J, Zhu L, Liu J, Zhu T, Xie Z et al. (2019). Metformin protects against oxidative stress injury induced by ischemia/ reperfusion via regulation of the lncRNA-H19/miR-148a3p/Rock2 axis. Oxidative Medicine and Cellular Longevity 2019: 1-18. DOI 10.1155/2019/8768327.

Zhang B, Zhang HX, Shi ST, Bai YL, Zhe X et al. (2019a). Interleukin11 treatment protected against cerebral ischemia/reperfusion injury. Biomedicine \& Pharmacotherapy 115: 108816. DOI 10.1016/j.biopha.2019.108816.

Zhang BF, Chen J, Jiang H (2019b). LncRNA H19 ameliorates myocardial ischemia-reperfusion injury by targeting miR22-3P. International Journal of Cardiology 278: 224. DOI 10.1016/j.ijcard.2018.11.017.

Zhang L, Li H, Zang Y, Wang F (2019c). NLRP3 inflammasome inactivation driven by miR-223-3p reduces tumor growth and increases anticancer immunity in breast cancer. Molecular Medicine Reports 19: 2180-2188. DOI 10.3892/mmr.2019.9889.

Zhang X, Li Y, Liang Y, Sun P, Wu X et al. (2017a). Distinguishing intracerebral hemorrhage from acute cerebral infarction through metabolomics. Revista de Investigación Clínica 69: 319-328. DOI 10.24875/RIC.17002348. 
Zhang X, Liu Q, Zhang C, Sheng J, Li S et al. (2019d). Puerarin prevents progression of experimental hypoxia-induced pulmonary hypertension via inhibition of autophagy. Journal of Pharmacological Sciences 141: 97-105. DOI 10.1016/j.jphs.2019.09.010.

Zhang X, Yan H, Yuan Y, Gao J, Shen Z et al. (2014). Cerebral ischemia-reperfusion-induced autophagy protects against neuronal injury by mitochondrial clearance. Autophagy 9: 1321-1333. DOI 10.4161/auto.25132.

Zhang Y, Qiao L, Xu W, Wang X, Li H et al. (2017b). Paeoniflorin attenuates cerebral ischemia-induced injury by regulating $\mathrm{Ca}(2+) / \mathrm{CaMKII} / \mathrm{CREB}$ signaling pathway. Molecules 22: 359. DOI 10.3390/molecules22030359.

Zhang Y, Yang X, Ge X, Zhang F (2019e). Puerarin attenuates neurological deficits via Bcl-2/Bax/cleaved caspase-3 and Sirt3/SOD2 apoptotic pathways in subarachnoid hemorrhage mice. Biomedicine \& Pharmacotherapy 109: 726-733. DOI 10.1016/j.biopha.2018.10.161.

Zhao GC, Yuan YL, Chai FR, Ji FJ (2017). Effect of Melilotus officinalis extract on the apoptosis of brain tissues by altering cerebral thrombosis and inflammatory mediators in acute cerebral ischemia. Biomedicine \& Pharmacotherapy 89: 1346-1352. DOI 10.1016/j.biopha.2017.02.109.

Zhao J, Wang H, Dong L, Sun S, Li L (2019). miRNA-20b inhibits cerebral ischemia-induced inflammation through targeting NLRP3. International Journal of Molecular Medicine 43: 1167-1178.

Zhao Y, Gan Y, Xu G, Hua K, Liu D (2020). Exosomes from MSCs overexpressing microRNA-223-3p attenuate cerebral ischemia through inhibiting microglial M1 polarization mediated inflammation. Life Sciences 260: 118403. DOI 10.1016/j.lfs.2020.118403.

Zhong Y, Yu C, Qin W (2019). LncRNA SNHG14 promotes inflammatory response induced by cerebral ischemia/ reperfusion injury through regulating miR-136-5p /ROCK1. Cancer Gene Therapy 26: 234-247. DOI 10.1038/s41417018-0067-5.

Zhou F, Wang L, Liu P, Hu W, Zhu X et al. (2014). Puerarin protects brain tissue against cerebral ischemia/reperfusion injury by inhibiting the inflammatory response. Neural Regeneration Research 9: 2074-2080. DOI 10.4103/16735374.147934 .

\section{Supplementary Figure}

A

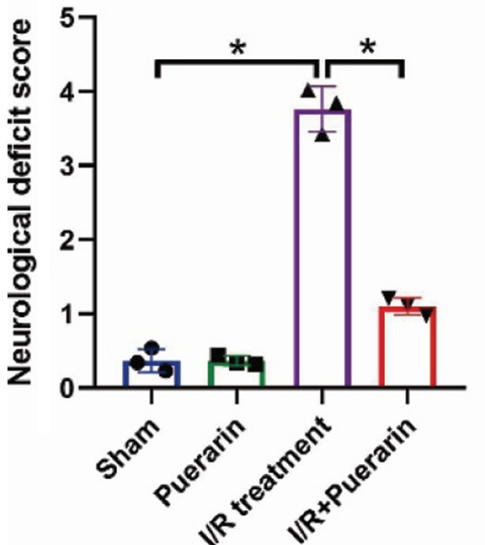

B

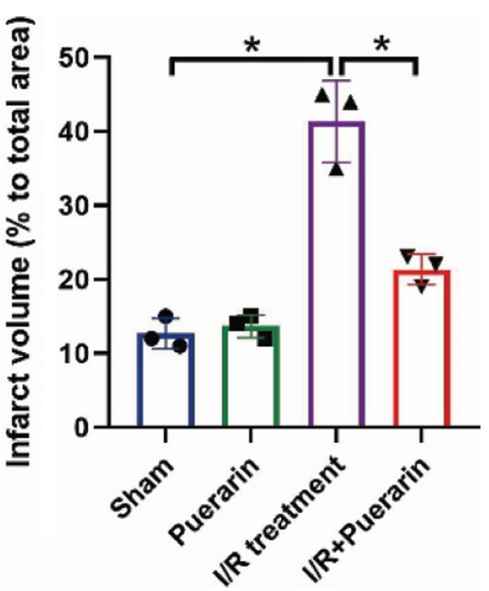

SUPPLEMENTARY FIGURE S1. The effects of Puerarin treatment on (A) the neurological deficit scores and (B) the volume of infarcted area in rat brain respectively by using the Longa scoring system and TTC staining assay. Each experiment was repeated three times; ${ }^{\star} P<0.05$. 